\title{
A Discotic Disguised as a Smectic: A Hybrid Columnar Bragg Glass
}

\author{
Karl Saunders, ${ }^{1}$ Leo Radzihovsky, ${ }^{2}$ and John Toner ${ }^{1}$ \\ ${ }^{1}$ Department of Physics, Materials Science Institute and Institute of Theoretical Science, University of Oregon, \\ Eugene, Oregon 97403 \\ ${ }^{2}$ Department of Physics, University of Colorado, Boulder, Colorado 80309
}

(Received 20 March 2000)

\begin{abstract}
We show that discotics, lying deep in the columnar phase, can exhibit an x-ray scattering pattern which mimics that of a somewhat unusual smectic liquid crystal. This exotic, new glassy phase of columnar liquid crystals, which we call a "hybrid columnar Bragg glass," can be achieved by confining a columnar liquid crystal in an anisotropic random environment of, e.g., strained aerogel. Long-ranged orientational order in this phase makes single-domain x-ray scattering possible, from which a wealth of information could be extracted. We give detailed quantitative predictions for the scattering pattern in addition to exponents characterizing anomalous elasticity of the system.
\end{abstract}

PACS numbers: 64.60.Fr, 61.30.Gd, 82.65.Dp

Until now, the x-ray scattering pattern given in Fig. 1 would be identified with a system in a somewhat unusual smectic phase with short-ranged translational and longranged orientational order within the smectic layers, i.e., a smectic composed of nematic, rather than liquid, layers. The set of on-axis quasisharp Bragg peaks along $q_{h}$ is a signature of the quasi-long-ranged translational order (i.e., the periodicity of the layering) that is characteristic of the bulk smectic phase. The presence of the other, broadened, peaks and the azimuthal anisotropy about the $q_{h}$ axis respectively indicate the incipient short-ranged translational order and the long-ranged orientational order within the smectic layers oriented perpendicular to $q_{h}$.

In this Letter we predict the existence of a remarkable new "hybrid columnar Bragg glass" (HCBG) phase [1,2] which, despite differing fundamentally from the smectic phase described above, shares the same qualitative scattering pattern illustrated in Fig. 1. Such mimickry of one phase by a completely different phase is unprecedented.

Columnar phases in pure, bulk (i.e., quenched-disorderfree) liquid crystals are phases that have long-ranged translational order in two directions, and short-ranged translational order (i.e., liquidlike correlations) in the third. I.e., they are regular two-dimensional lattices of onedimensional liquid columns [Fig. 2(a)]. In this Letter, we show that when such a system is confined in an anisotropic quenched random environment, e.g., strained aerogel [3], it becomes translationally disordered, but remains topologically ordered (i.e., free of topological defects such as dislocations). This novel state is the HCBG.

Like the smectic phase, the HCBG has translational order that is quasi-long-ranged in one direction and short ranged in another, as implied by Fig. 1. As illustrated in Fig. 2(b), the columns remain in roughly equidistant rows perpendicular to the stretching direction, but lose long-ranged translational order within each row. Nevertheless, the hexagonal orientational order is preserved, albeit uniaxially distorted due to the stretch. However, there are a number of fundamental differences between the two phases. First, unlike the smectic phase, the exponent $\eta_{\mathbf{G}}$ characterizing the shape of the quasi-long-ranged translational order peaks at $\mathbf{G}$, which are given by $I(\mathbf{q}) \propto$ $|\mathbf{q}-\mathbf{G}|^{-3+\eta_{G}}$, is independent of temperature. This property would allow the scattering patterns of the two phases to be distinguished through comparison of the line shapes as temperature is varied. Second, the correlations of the quasi-long-ranged order in the HCBG scale isotropically in space, in contrast to the well-known strongly anisotropic scaling of these correlations in the smectic. The third and most crucial difference between the two phases is their topological order, which distinguishes their elasticities but not their scattering. Specifically, the absence of the extra direction of long-ranged translational order, in the smectic phase, is caused by free topological defects, namely, unbound dislocations with Burgers vectors along the smectic planes. Although the HCBG also exhibits translational order that is quasi-long-ranged in one direction and short ranged in another, at long length scales it is distinguished from the smectic by being free of these unbound

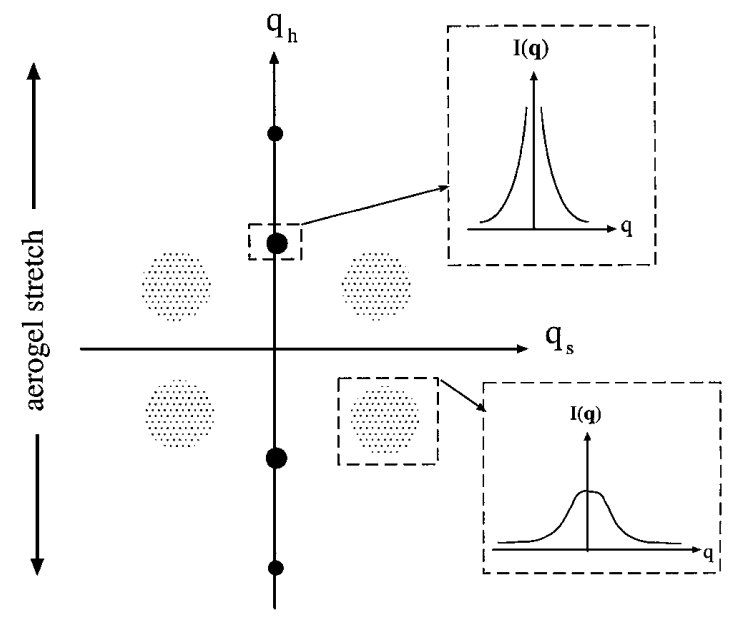

FIG. 1. X-ray scattering pattern in the $\perp$ plane for a class of hybrid columnar Bragg glass. 


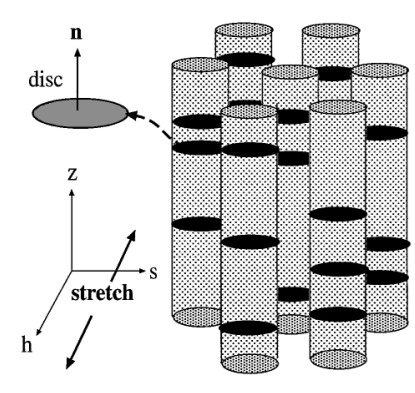

(a)

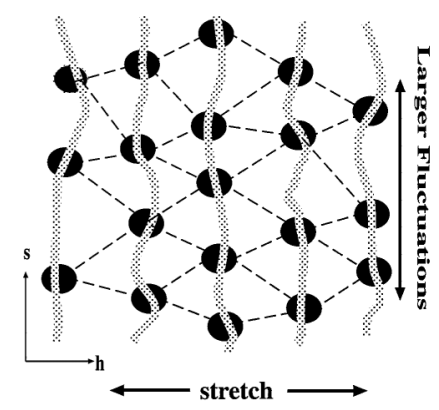

(b)
FIG. 2. (a) Alignment of the lattice for a uniaxial stretch. (b) Schematic of the distorted lattice in real space (top view).

dislocations, which would otherwise destroy the columnar phase topology of HCBG. One important experimental consequence of this absence of free dislocations is that the HCBG retains elastic resistance to distortions in the extra direction, albeit, as we discuss below, of a very strange, anomalous sort.

Of course, for sufficiently strong disorder free dislocations will eventually proliferate. The anisotropy, imposed by the strained aerogel, leads to the interesting possibility that dislocations with their Burgers vectors in the soft direction may unbind before those with their Burgers vectors in the hard direction, leading to the sequence of disorderdriven phase transitions HCBG $\rightarrow m=1$ smectic Bragg glass [4] $\rightarrow$ nematic elastic glass [5] with increasing aerogel density.

The rest of this Letter gives a more detailed theoretical description of the HCBG phase, including x-ray correlation lengths and universal exponents characterizing the anomalous elasticity. We relegate the technical details to a future publication [6].

Our model for a columnar phase consists of disk-shaped molecules with normals aligned along the $\hat{\mathbf{z}}$ direction. The disks form a hexagonal lattice in the $x y(\perp)$ plane and have liquidlike correlations along $\hat{\mathbf{z}}$, as illustrated in Fig. 2(a). We assume, and verify a posteriori, that despite considerable distortion, for sufficiently weak quenched disorder, the columnar phase topology is stable, i.e., our discotic liquid crystal remains free of unbound dislocations. Consequently this system can be described within an elastic theory, with a two-component ( $x$ and $y$ ) lattice site displacement vector $\mathbf{u}(\mathbf{r})$ and the discotic director $\hat{\mathbf{n}}(\mathbf{r})$ (the normal to the disks) as the only important long length-scale degrees of freedom. The disordering tendency of the aerogel is twofold: the strands act both to randomly pin the columnar lattice $[\mathbf{u}(\mathbf{r})]$ and to distort the orientations of the disk normals $[\hat{\mathbf{n}}(\mathbf{r})]$. Our starting Hamiltonian is that of a pure hexagonal discotic in isotropic aerogel,

$$
\begin{aligned}
H=\int_{\mathbf{r}} & {\left[\frac{B_{\perp}}{2}\left|\partial_{z} \mathbf{u}-\boldsymbol{\delta} \mathbf{n}\right|^{2}+\frac{1}{2} \lambda u_{i i}^{\perp} u_{j j}^{\perp}+\mu u_{i j}^{\perp} u_{i j}^{\perp}\right.} \\
& \left.+\operatorname{Re} \sum_{i} V_{i}(\mathbf{r}) e^{i \mathbf{G}_{i} \cdot \mathbf{u}(\mathbf{r})}-[\mathbf{g}(\mathbf{r}) \cdot \hat{\mathbf{n}}]^{2}\right]+H_{F}[\hat{\mathbf{n}}] .
\end{aligned}
$$

where $u_{i j}^{\perp}=\frac{1}{2}\left(\partial_{i}^{\perp} u_{j}+\partial_{j}^{\perp} u_{i}-\partial_{k} u_{i} \partial_{k} u_{j}\right)$ is the rotationally invariant symmetric strain tensor, $\delta \mathbf{n}(\mathbf{r}) \equiv$ $\hat{\mathbf{n}}(\mathbf{r})-\hat{\mathbf{z}}$, the $B_{\perp}$ term reflects the tendency of the molecular director (disk normal) $\hat{\mathbf{n}}(\mathbf{r})$ to lie along the local tangent $\hat{\mathbf{t}} \approx \hat{\mathbf{z}}+\partial_{z} \mathbf{u}$ to the liquidlike columns, $H_{F}[\hat{\mathbf{n}}]$ is the Frank free energy of the molecular directors, and $V_{i}(\mathbf{r})$ is a complex random pinning potential that couples to lattice site fluctuations along the reciprocal lattice basis vector, $\mathbf{G}_{i}$. At long length scales its correlations can be accurately represented as zero mean with short-ranged Gaussian statistics: $\overline{V_{i}(\mathbf{r}) V_{j}^{*}\left(\mathbf{r}^{\prime}\right)}=\tilde{\Delta}_{V} \delta_{i j}^{\perp} \delta^{d}\left(\mathbf{r}-\mathbf{r}^{\prime}\right)$ [5], where throughout this paper $\bar{x}$ denotes a quenched average over the disorder of the quantity $x$, while $\langle x\rangle$ denotes a thermal average. The last term describes the tendency of the disk normals $\hat{\mathbf{n}}(\mathbf{r})$ to align along the random local aerogel strand directed along $\mathbf{g}(\mathbf{r})$. This "random tilt" disorder is described by short-ranged isotropic correlations $\overline{g_{i}(\mathbf{r}) g_{j}\left(\mathbf{r}^{\prime}\right)}=1 / 2 \sqrt{\Delta} \delta_{i j} \delta^{d}\left(\mathbf{r}-\mathbf{r}^{\prime}\right)[5] . \Delta_{V}$ and $\Delta$ are phenomenological parameters which, in the simplest microscopic model, are proportional to the aerogel density, $\rho_{A}$. As for smectics [5], only these two types of disorder have important long distance effects.

A detailed analysis [5,6] has shown that this system exhibits a columnar Bragg glass phase with only short-ranged translational order. However, this changes if the aerogel is anisotropic. Aerogel anisotropy could be realized, e.g., by applying a strain to the strands. For heterotropic alignment between the disk normals and strands (assumed throughout), a uniaxial compression will lead to a phase in the same universality class as the Bragg glass phase of an Abrikosov flux lattice [1,2], with quasi-long-ranged translational order in both directions of the $\perp$ plane [6]. The more interesting HCBG phase with quasi-long-ranged order in only one $\perp$ direction can be obtained by applying a uniaxial stretch to the strands. For homeotropic alignment of strands and disk normals, the two phases reverse with respect to stretch and compression leaving all of our other predictions unchanged.

Uniaxial stretch to the aerogel strands (along $\hat{\mathbf{e}}_{h}$ ) causes the disk normals to align $\perp$ to this axis of stretch (Fig. 2). This can be accounted for by the addition of a term $\int_{\mathbf{r}} \gamma\left(\hat{\mathbf{e}}_{h} \cdot \hat{\mathbf{n}}\right)^{2}$ to the Hamiltonian, where $\gamma$ is a phenomenological parameter, which we expect to be a monotonically increasing function of $\rho_{A}$ and strain. The uniaxial stretch also breaks both the hexagonal symmetry of the lattice and its rotation invariance. Thus, we are forced to consider both a more general lattice structure and an elastic Hamiltonian that is not invariant under rotations of the lattice. We can take into account both of these effects by replacing the harmonic pieces of the hexagonal elastic energy $\int_{\mathbf{r}}\left(\frac{1}{2} \lambda u_{i i}^{\perp} u_{j j}^{\perp}+\mu u_{i j}^{\perp} u_{i j}^{\perp}\right)$ with the more general harmonic elastic energy $\frac{1}{2} \int_{\mathbf{r}} C_{i j k l} \partial_{i}^{\perp} u_{j} \partial_{k}^{\perp} u_{l}$, where the elastic constant tensor $C_{i j k l}$ is not symmetric under interchange of its first two or second two indices, due to the lack of in-plane rotation invariance just discussed. The terms cubic and quartic in $\mathbf{u}$ in (1) must also 
be so generalized, of course. Because of the $B_{\perp}$ term in Eq. (1), fluctuations of $\hat{\mathbf{n}}$ from the local column tangent $\hat{\mathbf{t}}$ are small, i.e., $\delta \mathbf{n} \approx \partial_{z} \mathbf{u}$.

We have analyzed [6] this generalization of the model [Eq. (1)] by using renormalization group (RG) methods [5]. One of the most surprising conclusions of this analysis is that, at long length scales, fluctuations $u_{h} \equiv \hat{\mathbf{e}}_{h} \cdot \mathbf{u}$ along the direction of stretch decouple from those $u_{s} \equiv$ $\hat{\mathbf{e}}_{s} \cdot \mathbf{u}$ orthogonal to this direction, where we have denoted the axis of stretch, $\hat{\mathbf{e}}_{h}$, as "hard" $(h)$ and called the other $\perp$ axis, orthogonal to $\hat{\mathbf{e}}_{h}$ "soft" $(s)$, i.e., $\mathbf{r}_{\perp}=$ $\left(r_{h}, r_{s}\right)$. That is, all couplings between $u_{s}$ and $u_{h}$ that are present in the full elastic tensor $C_{i j k l}$ are effectively irrelevant, leaving $C_{i j k l}$ in the form $C_{i j k l}=B_{s s} \delta_{i s} \delta_{j s} \delta_{k s} \delta_{l s}+$ $B_{s h} \delta_{i s} \delta_{j h} \delta_{k s} \delta_{l h}+B_{h s} \delta_{i h} \delta_{j s} \delta_{k h} \delta_{l s}+B_{h h} \delta_{i h} \delta_{j h} \delta_{k h} \delta_{l h}$.

The total Hamiltonian for the system can therefore be expressed as a sum of decoupled Hamiltonians for $u_{h}$ and $u_{s}: H_{\text {tot }}\left[u_{h}, u_{s}\right]=H_{X Y}\left[u_{h}\right]+H_{m=1}\left[u_{s}\right]$, with

$$
\begin{aligned}
H_{X Y}=\frac{1}{2} \int_{\mathbf{r}} & {\left[\gamma\left|\partial_{z} u_{h}\right|^{2}+B_{s h}\left|\partial_{s} u_{h}\right|^{2}+B_{h h}\left|\partial_{h} u_{h}\right|^{2}\right.} \\
& \left.+\mathcal{R} e \sum_{i} V_{i}(\mathbf{r}) e^{i\left(\mathbf{G}_{i} \cdot \hat{\mathbf{e}}_{h}\right) u_{h}(\mathbf{r})}\right], \\
H_{m=1}=\int_{\mathbf{r}}\{ & \frac{K}{2}\left(\partial_{z}^{2} u_{s}\right)^{2}+B_{s s}\left[\partial_{s} u_{s}-\frac{1}{2}\left(\partial_{z} u_{s}\right)^{2}\right]^{2} \\
+ & \left.B_{h s}\left(\partial_{h} u_{s}\right)^{2}-g_{z}\left(\mathbf{g} \cdot \hat{\mathbf{e}}_{s}\right)\left(\partial_{z} u_{s}\right)\right\} \cdot
\end{aligned}
$$

These two parts of $H_{\text {tot }}$, which describe the fluctuations of the hard and soft phonon fields $u_{h}$ and $u_{s}$, are not new. Hamiltonians of precisely this form have been previously used to describe the random field $X Y$ model [2] and the " $m=1$ smectic Bragg glass" [4], respectively, and have been studied extensively [1]. However, a columnar phase confined in anisotropic aerogel, whose Hamiltonian, $H_{\text {tot }}$, is a combination, or hybrid, of the two, is entirely novel.

The $u_{h}$ fluctuations of our system are the same as those of a random field $X Y$ model (with anisotropic stiffness) and are given by $\overline{\left\langle\left(u_{h}(\mathbf{r})-u_{h}(\mathbf{0})\right)^{2}\right\rangle}=C(d) \ln r / G_{0 h}^{2}$, where $G_{0 h}$ is the lattice spacing of the projection of the discotic reciprocal lattice onto the hard axis. They diverge logarithmically as a function of distance, implying that the translational order along the hard direction is quasilong-ranged. While these elastic distortions are reminiscent of the famous Landau-Peierls $\ln r$ fluctuations of bulk smectics, they differ crucially in two ways. Firstly, they are disorder, rather than thermally, driven with $C(d)$ universal $[C(3) \approx 1.1]$ and the logarithm persisting in all $2<d<4$. Secondly, they are isotropic in their scaling. In contrast, in a bulk smectic the layer fluctuations within the layers scale differently from those along the normal to the layers.

In $H_{m=1}$, the combination of relevant anharmonic terms and large disorder-induced $u_{s}$ fluctuations leads to strong anomalous elasticity $[5,6]$. By anomalous elasticity we mean that the full, anharmonic theory with constant $K$,
$B_{s s}$, and $\Delta$ can, at small wave vector $k \ll \xi_{\mathrm{NL}}^{-1}$ (where $\xi_{\mathrm{NL}}$ is a nonuniversal length determined by material parameters, e.g., aerogel density), be effectively replaced by a harmonic theory with wave vector dependent $K, B_{s s}$, and $\Delta$, given by

$$
\begin{aligned}
K(\mathbf{k}) & =K k_{z}^{-\eta_{K}} f_{K}\left(k_{h} / k_{z}^{\zeta_{h}}, k_{s} / k_{z}^{\zeta_{s}}\right), \\
B_{s s}(\mathbf{k}) & =B_{s s} k_{z}^{\eta_{B}} f_{B}\left(k_{h} / k_{z}^{\zeta_{h}}, k_{s} / k_{z}^{\zeta_{s}}\right), \\
\Delta(\mathbf{k}) & =\Delta k_{z}^{-\eta_{\Delta}} f_{\Delta}\left(k_{h} / k_{z}^{\zeta_{h}}, k_{s} / k_{z}^{\zeta_{s}}\right) .
\end{aligned}
$$

$B_{h s}$ is not significantly renormalized, that is, $B_{h s}(\mathbf{k})=B_{h s}$, independent of wave vector. Here the anisotropy exponents $\zeta_{s} \equiv 2-\left(\eta_{B}+\eta_{K}\right) / 2$ and $\zeta_{h} \equiv 2-\eta_{K} / 2$. The exponents, evaluated using the RG and a high precision $\epsilon$-expansion were found to be $\eta_{K}=0.50, \eta_{B}=0.26$, and $\eta_{\Delta}=0.13[4,6]$. We also predict that the anomalous exponents will obey the following exact scaling relation in $d=3$ :

$$
1+\eta_{\Delta}=\eta_{B} / 2+2 \eta_{K} .
$$

The translational order of the system along the soft direction is short ranged and is characterized by the algebraic and anisotropic divergence of $u_{s}$ correlations,

$$
\overline{\left\langle\left(\delta u_{s}(\mathbf{r})\right)^{2}\right\rangle}= \begin{cases}\left(\frac{K}{B_{s s}}\right)\left(\frac{r_{z}}{\xi_{z}}\right)^{\chi_{z}}, & r_{z} \gg r_{s, h}, \\ \left(\frac{K}{B_{s s}}\right)\left[\frac{r_{s}}{\xi_{z}^{2}}\left(\frac{K}{B_{s s}}\right)^{1 / 2}\right]^{\chi_{s},}, & r_{s} \gg r_{h, z}, \\ \left(\frac{K}{B_{s s}}\right)\left[\frac{r_{h}}{\xi_{z}^{2}}\left(\frac{K}{B_{s h}}\right)^{1 / 2}\right]^{\chi_{h}}, & r_{h} \gg r_{s, z},\end{cases}
$$

where we have defined $\delta u_{s}(\mathbf{r}) \equiv u_{s}(\mathbf{r})-u_{s}(\mathbf{0}), \chi_{z} \equiv$ $1-\eta_{K}+\eta_{B} / 2+\eta_{\Delta}=\eta_{B}+\eta_{K}, \quad \chi_{s, h} \equiv \chi_{z} / \zeta_{s, h}$, and $\xi_{z} \equiv K^{2} B_{s h}^{1 / 2} /\left(\Delta B_{s s}^{1 / 2}\right)$. The exact scaling relation (4) could be experimentally tested by using the more general expressions for $\chi_{z}, \chi_{s}, \chi_{h}$ in terms of all three exponents, and verifying that $\eta_{B}, \eta_{K}$, and $\eta_{\Delta}$ obey the relation (4). Our $\epsilon$-expansion results for the $\eta$ 's imply $\chi_{z}=0.76$, $\chi_{s}=0.47$, and $\chi_{h}=0.43$. The fluctuations given in Eq. (5), like those along the hard direction, are disorder, rather than thermally driven.

Despite this lack of translational order, our detailed calculations [6] indicate that dislocation loops remain bound for weak disorder, and therefore the low temperature phase replacing the columnar phase must be distinct from the smectic and hexatic, separated from them by a thermodynamically sharp dislocation unbinding phase transition.

The stability of this exotic glass phase is contingent upon our assumption of long-ranged orientational order. We validate this assumption by calculating $\overline{\left\langle|\mathbf{n}(\mathbf{r})-\mathbf{n}(\mathbf{0})|^{2}\right\rangle}=$ $\overline{\left\langle\left|\partial_{z} \mathbf{u}(\mathbf{r})-\partial_{z} \mathbf{u}(\mathbf{0})\right|^{2}\right\rangle}$ and showing that it does not diverge as $\mathbf{r} \rightarrow \infty$ [6]. Although equilibration into the ground state might be slow and therefore require field alignment, this orientational order would allow experimentalists to investigate single-domain samples of HCBG. The anisotropic scaling information which is usually lost in a powder averaged x-ray scattering experiment would be retained in a single-domain experiment, allowing detailed tests of our predictions for $\eta_{K}, \eta_{B}$, and $\eta_{\Delta}$. 
The scattering pattern in the $\perp$ plane, obtained from a single-domain sample, would consist of a set of spots rather than the set of rings that one would expect from a powder sample. This pattern depends crucially on the relative orientations within the $\perp$ plane of the reciprocal lattice and the axis of stretch, $\hat{\mathbf{e}}_{h}$, which could vary from discotic to discotic since it depends on the microscopic interactions between the disks and strands. The intensity of a Bragg spot at a reciprocal lattice vector $\mathbf{G}$ is

$$
\begin{aligned}
I(\mathbf{G}) \propto & \int_{\mathbf{r}} \exp \left\{-\overline{\left\langle[\mathbf{G} \cdot(\mathbf{u}(\mathbf{r})-\mathbf{u}(\mathbf{0}))]^{2}\right\rangle} / 2\right\} \\
\propto & \int_{\mathbf{r}} \exp \left\{-\left[G_{h}^{2} \overline{\left\langle\left(u_{h}(\mathbf{r})-u_{h}(\mathbf{0})\right)^{2}\right\rangle}\right.\right. \\
& \left.\left.+G_{s}^{2} \overline{\left\langle\left(u_{s}(\mathbf{r})-u_{s}(\mathbf{0})\right)^{2}\right\rangle}\right] / 2\right\} .
\end{aligned}
$$

Unless $G_{s}=0$, the algebraically diverging $u_{s}$ fluctuations dominate the logarithmically diverging $u_{h}$ fluctuations and the integrand is stretched-exponentially damped, leading to an anisotropically broadened Bragg peak. If, however, $G_{s}=0$, then the exponential becomes $r^{-0.55 n^{2}}$, where $n=G_{h} / G_{0 h}$, with $G_{0 h}$ being the magnitude of the smallest $\mathbf{G}$ lying on the hard axis, and for $n<3$ the integral diverges as $r \rightarrow \infty$, leading to quasisharp peaks for those $n$ 's. We therefore predict two classes of hybrid columnar Bragg glasses. The first, which we call a commensurate HCBG, has some reciprocal lattice vectors that lie along the hard axis, and will exhibit a scattering pattern with peaks lying on the hard axis, with the first two quasisharp. In the second, incommensurate HCBG, class, all of the peaks lie off the hard axis, and are anisotropically broadened by the contribution from the $u_{s}$ fluctuations. The smecticlike scattering pattern (Fig. 1), with a quasisharp peak on the first ring, will therefore be observed only for commensurate HCBG's.

The dependence of the anisotropically broadened peak widths on the bare elastic constants is the same for both

classes of HCBG. Setting the $u-u$ correlation functions [given in Eq. (5)] equal to $G_{s}^{-2}$, and solving for $\left(r_{z}=\right.$ $\left.\xi_{z}^{X}\right)^{-1},\left(r_{s}=\xi_{s}^{X}\right)^{-1}$, and $\left(r_{h}=\xi_{h}^{X}\right)^{-1}$ gives the width of the peak at $\mathbf{G}$ along the $z, s$, and $h$ directions:

$$
\begin{gathered}
\left(\xi_{z}^{X}\right)^{-1}=\xi_{z}^{-1}\left(G_{s}^{2} K / B_{s s}\right)^{\chi_{z}^{-1}}, \\
\left(\xi_{s}^{X}\right)^{-1}=\xi_{z}^{-2}\left(K / B_{s s}\right)^{1 / 2}\left(G_{s}^{2} K / B_{s s}\right)^{\chi_{s}^{-1}}, \\
\left(\xi_{h}^{X}\right)^{-1}=\xi_{z}^{-2}\left(K / B_{s h}\right)^{1 / 2}\left(G_{s}^{2} K / B_{s s}\right)^{\chi_{h}^{-1}} .
\end{gathered}
$$

The temperature dependence of $\xi_{z, s, h}^{X}$ could be used to determine the exponents $\eta_{K}, \eta_{B}$ and $\eta_{\Delta}$ since the bulk $K(T), B_{s s}(T)$, and $B_{s h}(T)$ in Eqs. (7a)-(7c) have $T$ dependences that can be extracted from data on bulk materials. A more direct way to observe the anomalous elasticity would be a measurement of the $u-u$ correlation function $I(\mathbf{q}) \propto \overline{\left\langle\left|u_{S}(\delta \mathbf{q})\right|^{2}\right\rangle}$, which can be obtained [6] for large q (i.e., q's with at least one component bigger than the corresponding inverse x-ray correlation length quoted above) by looking at an intermediate regime in the "tails" of the broad x-ray scattering peaks. In those tails, [i.e., for $\mathbf{q}=\mathbf{G}+\delta \mathbf{q}$ with $\xi_{\mathrm{NL}}^{-1} \gg\left|\delta q_{\alpha}\right| \gg\left(\xi_{\alpha}^{\chi}\right)^{-1}$ for at least one Cartesian direction $\alpha=(h, s, z)$ [7] ], it can be shown that

$$
I(\mathbf{q}) \propto \frac{\Delta(\delta \mathbf{q}) q_{z}^{2}}{\left[B_{s s}(\delta \mathbf{q}) \delta q_{s}^{2}+K(\delta \mathbf{q}) \delta q_{z}^{4}+B_{h s} \delta q_{h}^{2}\right]^{2}} .
$$

Hence, the $\mathbf{q}$ dependence of $B_{s s}(\mathbf{q}), K(\mathbf{q})$, and $\Delta(\mathbf{q})$ in Eqs. (3a) and (3b) could be tested directly by a fit of scattering data to these tails.

A related experimental approach, which has the advantage of not being restricted to wave vectors larger than the inverse x-ray correlation lengths, but can, rather, explore arbitrarily small q's, is light scattering, which measures director fluctuations. These can be related to the $u-u$ correlations via our condition $\delta \mathbf{n} \approx \partial_{z} \mathbf{u}$. This yields

$$
\overline{\left\langle\left|\delta n_{s}(\mathbf{q})\right|^{2}\right\rangle}=\frac{\Delta(\mathbf{q}) q_{z}^{4}}{\left[B_{s s}(\mathbf{q}) q_{s}^{2}+K(\mathbf{q}) q_{z}^{4}+B_{h s} q_{h}^{2}\right]^{2}},
$$

$$
\overline{\left\langle\left|\delta n_{h}(\mathbf{q})\right|^{2}\right\rangle}= \begin{cases}{\left[C(3) / 2 G_{0 h}^{2}\right]\left(q_{z}^{2} / q^{3}\right),} & \text { commensurate } \\ k_{B} T q_{z}^{2} G(\mathbf{q})+\Delta_{h}(\mathbf{q}) q_{z}^{4} G(\mathbf{q})^{2}, & \text { incommensurate }\end{cases}
$$

where $\Delta_{h}(\mathbf{q})$ in Eq. (9b) is a renormalized $\mathbf{q}$-dependent tilt disorder variance obeying the scaling law $\Delta_{h}(\mathbf{q})=\Delta_{h} q_{z}^{-\left(\eta_{\Delta}+\eta_{B}\right)} f_{\Delta_{h}}\left(q_{h} / q_{z}^{\zeta_{h}}, q_{s} / q_{z}^{\zeta_{s}}\right)$ and $G(\mathbf{q})=$ $1 /\left(B_{s h} q_{s}^{2}+\gamma q_{z}^{2}+B_{h h} q_{h}^{2}\right)$. The commensurate and incommensurate cases differ because in the commensurate case there is a random field acting on $u_{h}$, while in the incommensurate case there is no random field, leaving the random tilt as the dominant disorder.

L. R. acknowledges support by the NSF DMR-9625111, the MRSEC DMR-9809555, and the Sloan and Packard Foundations. J. T. and K. S. were supported by the NSF DMR-9634596 and DMR-9980123.

[1] For a general discussion of "Bragg glass" phases see, e.g., T. Nattermann and S. Scheidl, Adv. Phys. 49, 607 (2000).
[2] T. Giamarchi and P. Le Doussal, Phys. Rev. Lett. 72, 1530 (1994); D. S. Fisher, Phys. Rev. Lett. 78, 1964 (1997).

[3] There has been an immense amount of excellent work on liquid crystals in aerogel. See, e.g., Liquid Crystals in Complex Geometries, edited by G. P. Crawford and S. Zumer (Taylor \& Francis, London, 1996).

[4] B. Jacobsen, K. Saunders, L. Radzihovsky, and J. Toner, Phys. Rev. Lett. 83, 1363 (1999).

[5] L. Radzihovsky and J. Toner, Phys. Rev. B 60, 206 (1999); Phys. Rev. Lett. 79, 4214 (1997).

[6] K. Saunders, L. Radzihovsky, and J. Toner (unpublished).

[7] We have shown in [6] that the ratio $\xi_{\mathrm{NL}} / \xi_{\alpha}^{\chi} \rightarrow 0$ as the aerogel density goes to zero. Hence, one could make the width of this window in $\delta q_{\alpha}$ as large as one likes simply by making the aerogel density sufficiently small. 